\title{
Surgical Aspect of Pancreas Transplantation
}

\author{
Duck-Jong Han and Takashi Kenmochi
}

\section{The Donor}

See the Living Donor chapter.

\section{Recipient}

See the Living Donor chapter.

D.-J. Han $(\bowtie)$

Asan Medical Center, Seoul, Korea

T. Kenmochi

Fujita Health University, Toyoake, Japan

e-mail: kenmochi@fujita-hu.ac.jp 